\title{
Evidence for size-dependent mechanical properties from simulations of nanoscopic polymeric structures
}

\author{
Thomas R. Böhme and Juan J. de Pablo \\ Department of Chemical Engineering, University of Wisconsin-Madison, Madison, Wisconsin 53706
}

(Received 7 November 2001; accepted 13 March 2002)

\begin{abstract}
Discontinuous molecular dynamics simulations of a model polymer have been conducted to investigate the glass transition of ultrathin films and the mechanical properties of nanoscopic structures. Continuum mechanics models have been applied to interpret simulation data and extract apparent Young's Moduli. Consistent with experiments, the results of simulations indicate that the glass transition temperature of thin films can be higher or lower than that of the bulk, depending on the nature of polymer-substrate interactions. Simulations also indicate that the mechanical properties of nanoscopic structures can be considerably different from those of the bulk. An analysis of molecular strain distributions in nanostructures undergoing a deformation indicate that significant stress relaxation occurs at air-polymer interfaces. A comparison of these distributions to the results of continuum, finite-element calculations reveal pronounced differences between the continuum and molecular approaches. (C) 2002 American Institute of Physics. [DOI: 10.1063/1.1476315]
\end{abstract}

\section{INTRODUCTION}

The current roadmap for semiconductor fabrication envisions large-scale production of nanoscopic structures, with critical dimensions below $100 \mathrm{~nm}$, in the near future. ${ }^{1}$ The term "structure" is used in this work to denote a geometrical construct, such as a "beam," a "wall," or a "plate" made out of polymer molecules. These structures are fabricated by lithographic processes, which generally involve a "wet" polymer dissolution step followed by rinsing and drying. It has recently become apparent that the collapse of small polymer structures during rinsing and drying threatens to become one of the major obstacles to attain this goal. ${ }^{2}$ This collapse has been attributed to capillary forces caused by the surface tension of the rinse liquid. ${ }^{2,3}$ Simple continuum-mechanics models of cantilever beams have been used to describe the collapse of polymer structures such as "walls" or "plates." 2,3 These models (and many other aspects of lithography and nanofabrication) implicitly assume the validity of continuum mechanics, even for structure sizes well below $100 \mathrm{~nm}$.

However, as the dimensions decrease and become comparable to molecular length scales, the assumption of a continuum could be called into question. This is particularly relevant for polymeric materials, where an individual molecule can measure tens of nanometers. In recent years, various attempts have been made to quantify the elastic constants of small systems. ${ }^{4-6}$ However, to the best of our knowledge, experimental data are not available for the Young's Modulus of polymeric structures below dimensions of $100 \mathrm{~nm}$. The characterization of such small structures is challenging, and molecular simulations could provide valuable insights to guide, supplement, or help interpret laboratory experiments.

There is a growing awareness that, in ultrasmall or confined systems, several material properties exhibit departures from bulk behavior. While still a matter of debate, it is increasingly recognized, for example, that the glass transition temperature of ultrathin polymeric films can be significantly different from that of the bulk. ${ }^{7-11}$ This change in the glass transition temperature has been linked to a change of the density and segmental mobility near a substrate or an interface. ${ }^{12-14}$ Since density and segmental mobility are important factors in the mechanical behavior of a material, it is of interest to investigate if (and to what extent) the mechanical properties of materials in nanoscopic structures also exhibit departures from bulk behavior.

In this work we have used Discontinuous Molecular Dynamics (DMD) simulations to determine the glass transition temperature $\left(T_{g}\right)$ of thin polymer films and to explore the mechanical behavior of nanoscopic structures as a function of size. The methods and models employed in this work have been used in the past to address various questions in polymer physics, ${ }^{15,16}$ including the thickness dependence of $T_{g} \cdot{ }^{12}$ When applied to the study of nanoscopic "plates" or "lines," these methods predict a pronounced drop of the Young's modulus at nanoscopic length scales.

Only a handful of experimental techniques are available for manufacture of ultrasmall, nanoscopic polymeric structures. The thermophysical properties of the material in such small structures are likely to be highly sensitive to the particular details of a manufacturing technique. In this work, three different preparation methods were used to generate the simulated structures, thereby providing a means to investigate the dependence of the mechanical properties on the history of the sample. We find that, in general, the mechanical properties of polymeric nanoscopic structures differ considerably from those of the bulk.

The remainder of this work is organized as follows. Section II provides a brief description of the molecular model and the simulation technique. Section III discusses the simulation of thin polymeric films and the determination of an apparent glass transition temperature. Section IV discusses the preparation of polymeric nanoscopic structures, and the 
simulation of their mechanical properties. Our results are discussed in Sec. V, where an analysis of plastic deformation and the resulting molecular strains is also presented. We conclude with a brief summary of our findings.

\section{MOLECULAR MODEL}

The discontinuous polymer model adopted in this work consists of spherical interaction sites connected by flexible strings. This model can only provide a coarse-grained, minimalistic description of an unentangled linear polymer. While more realistic representations could be used to examine the mechanical properties of nanoscopic structures, the calculations would be considerably more demanding. The fundamental question addressed here is simply whether a glassy, amorphous polymeric material exhibits the same elastic modulus in a small nanoscopic structure than in the bulk; given the exploratory nature of this first study, the use of a more refined model is not warranted at this point.

The simulations of nanoscopic structures reported in this work were conducted using a Discontinuous Molecular Dynamics (DMD) technique. This method, developed by Alder and Wainwright, ${ }^{17}$ was first used for polymeric systems by Rapaport. ${ }^{18}$ Details of its implementation have been thoroughly discussed by Smith. ${ }^{19}$ The published algorithms were modified slightly in this work to allow for the application of external forces to the particles.

In DMD simulations, particles interact via a pairwise additive square-well potential, which depends on the distance $r$ between two interaction sites. The potential energy of interaction between any two such sites is given by

$$
U(r)=\left\{\begin{array}{cc}
\infty & \text { for } r \leqslant \sigma, \\
-\epsilon & \text { for } \sigma<r \leqslant \sigma_{2}, \\
0 & \text { for } r>\sigma_{2},
\end{array}\right.
$$

where $\sigma$ denotes the diameter of the particles, and where $\sigma_{2}-\sigma$ represents the range of attraction between them.

Polymer molecules are represented as linear chains of $N$ freely jointed square-well interaction sites. All of the simulations reported here were conducted with molecules of 16 interaction sites. The potential energy of interaction between bonded sites is given by

$$
U(r)=\left\{\begin{array}{cc}
\infty & \text { for } r \leqslant \sigma, \\
0 & \text { for } \sigma<r \leqslant \sigma+\delta, \\
\infty & \text { for } r>\sigma+\delta,
\end{array}\right.
$$

where $\delta$ represents the maximum bond length between two adjacent sites.

For all the simulations presented here, the dimensionless parameters that characterize these interactions were set to $\sigma=1.0, \sigma_{2}=1.5, \epsilon=1.0$, and $\delta=0.025 \sigma$. Unless otherwise specified, all the results presented in this work are given in reduced units, which are denoted by an asterisk.

A DMD algorithm can be implemented efficiently on current desktop computers. Our calculations indicate that the use of a discontinuous potential (and a DMD method) leads to gains of about one order of magnitude in computer time over conventional molecular dynamics simulations on con- tinuous (e.g., Lennard-Jones-type) potentials. This high efficiency permits simulation of relatively large systems over time scales of microseconds.

\section{GLASS TRANSITION TEMPERATURE OF THIN FILMS}

The starting geometry for most of our simulations is a thin polymer film. We begin this work by examining how the glass transition temperature $\left(T_{g}\right)$ changes in thin films. A study of $T_{g}$ using the same model was published recently, but few details were provided regarding the actual calculations; ${ }^{12}$ a more extended discussion is presented here.

\section{A. Film preparation}

The properties of glasses are highly sensitive to thermal history; the generation of an initial configuration plays an important role on the outcome of our simulations. The annealing process and the geometries considered in our work are described in this section. Four types of boundary conditions were considered: free-standing films, films supported on an attractive substrate, and films grafted to a surface according to two distinct prescriptions.

\section{Free-standing films}

Free-standing films were prepared by creating a random, low-density configuration in a rectangular simulation box of dimensions $20 \sigma \cdot 20 \sigma \cdot 60 \sigma$. Those configurations consisted of 200-800 polymer chains, depending on the film thickness. Periodic boundary conditions were applied in all three directions. The systems were then allowed to equilibrate at a temperature of $T^{*}=1.0$, which is well in the liquid regime (the estimated glass transition temperature is approximately $T_{g} \approx 0.33$ ). During this period, the systems self-assembled into thin films in order to minimize the surface area. After equilibrating the films at a temperature of $T^{*}=1.0$, they were cooled down to a temperature of $T^{*}=0.62$, which was the starting point for the final production runs. At this temperature, the films were simulated for at least five times the characteristic relaxation time of the bond-bond autocorrelation function, $\tau_{\text {bond }}^{*}$, to ensure thoroughly equilibrated initial configurations. For free-standing films at this temperature we find $\tau_{\text {bond }}^{*} \approx 320$.

\section{Supported films}

Supported films were prepared in a manner similar to that employed for free-standing films. Instead of applying periodic boundary conditions in all three dimensions, a smooth square-well wall was introduced to represent a substrate. The interaction between the wall and the polymer particles was characterized by $\sigma_{\text {wall }}=1.0, \sigma_{2, \text { wall }}=1.5$, and $\epsilon_{\text {wall }}=2.0$, i.e., the interaction with the wall was twice as strong as that between polymer particles. During the initial equilibration stage at $T^{*}=1.0$, films showed a tendency to form in the middle of the box (rather than near the wall). They were therefore shifted repeatedly towards the wall in order for the substrate to influence the film formation process. After the films were deposited on the substrate, they were cooled down to $T^{*}=0.62$ and equilibrated for at least $t^{*}=5 \tau_{\text {bond }}^{*}$. 


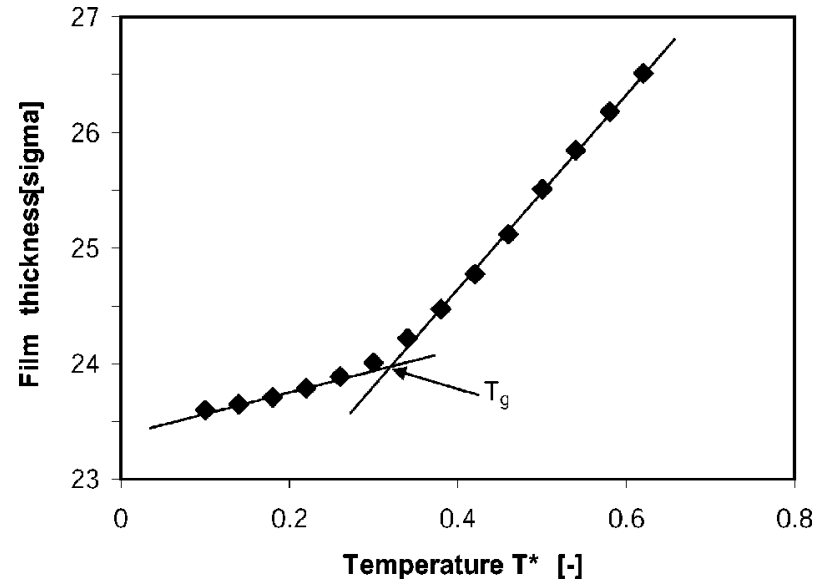

FIG. 1. Film thickness as a function of temperature for a free-standing polymer film.

\section{Grafted films}

Grafted films were generated from supported films at $T^{*}=1.0$. The interaction with the substrate was chosen to be only half the strength of the particle-particle interactions. To model the grafting of the polymer to the substrate, during the equilibration process at $T^{*}=1.0$ some of the monomers were attached to the wall using flexible bonds. The interaction potential for polymer-substrate bonds was the same as that between adjacent particles in a polymer chain [Eq. (2)]. The grafting process was conducted by creating a bond between the substrate and all the particles that collided with it during the simulation run until the desired grafting density was achieved.

Two types of grafted films were considered. In the first, 125 interaction sites on an area of $20 \sigma \cdot 20 \sigma$ were bonded to the surface. In the second, only chain ends were grafted. In this case, the number of grafted particles was 50. After completion of the grafting process, the films were cooled to $T^{*}=0.62$ and equilibrated for $t^{*}=5 \tau_{\text {bond }}^{*}$.

\section{B. Determination of the glass transition temperature}

An apparent $T_{g}$ can be extracted from simulations by observing how the density of the polymer changes with temperature during cooling. The film thickness as a function of temperature is shown in Fig. 1 for a free-standing film of approximately $h \approx 25 \sigma$. The graph shows a distinct change of the thermal expansion coefficient around $T^{*} \approx 0.33$. We identified $T_{g}$ as the intersection of two linear fits through the four lowest temperature data points and the five highest temperature data points.

Films prepared as outlined above provided the starting point for annealing calculations. Several simulation runs were performed by decreasing the temperature in a stepwise manner, starting from $T^{*}=0.62$. The temperature was lowered in decrements of $\Delta T^{*}=-0.04$, down to $T^{*}=0.10$. At each temperature, the films were simulated for $2 \cdot 10^{8}$ collision events (which is equivalent to $t^{*}=600$ for a thin film). The first half of each simulation run was discarded; only data from the second half of each run were used for statistical analysis at any given temperature. The simulation runs were

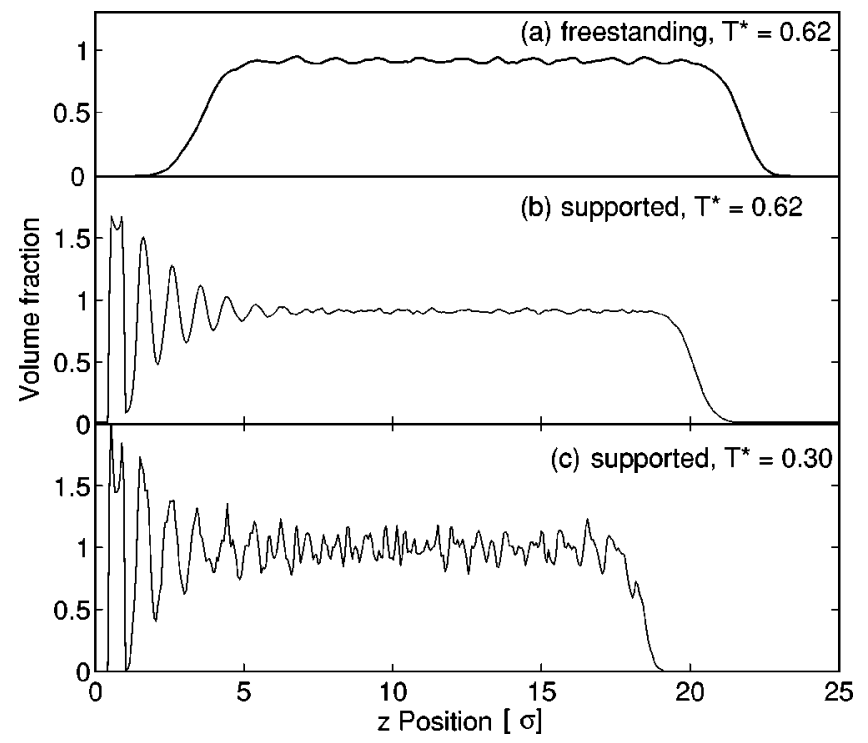

FIG. 2. Density profiles in the direction normal to the free surfaces for (a) free-standing film at $T^{*}=0.62$, (b) supported film at $T^{*}=0.62$, and (c) supported film at $T^{*}=0.30$.

as long as feasible within our computational resources. At a given temperature, a simulation of a film of $25 \sigma$ thickness required approximately $50 \mathrm{~h}$ of CPU time on a current desktop computer. Here we emphasize that, near $T_{g}$, relaxation times increase by several orders of magnitude and can exceed several seconds or minutes. Unfortunately, time scales beyond hundreds of microseconds are currently inaccessible to molecular simulations; the results presented here should therefore be viewed as an upper bound to $T_{g}$.

For each simulation run, a density profile normal to the plane of the film was calculated. Typical profiles for freestanding and supported films are shown in Fig. 2. Determining the thickness of the films from the density profile in a systematic manner poses several difficulties. First, the decay from the average density to zero is not sharp but gradual, and the boundaries of the films are not clearly defined. Second, the density profiles can be noisy and exhibit kinks and other irregularities. As can be seen in Fig. 2(c), this is particularly true for temperatures around and below $T_{g}$, where local density inhomogeneities are frozen in and do not average out, even after long simulation times.

It is therefore important to employ a method that is not too sensitive to such disturbances, but still delivers the high accuracy needed for systematic determination of $T_{g}$. As a first step, an average density $\rho_{0}$ was calculated over the entire film. Then, a new average $\rho_{1}$ was calculated using only the inner region of the film, which was bounded by the first and last point where the density exceeded the average $\rho_{0}$ calculated before. From this average $\rho_{1}$, a thickness was calculated by assuming a uniform density across the whole film. This method gave results that were largely independent of the bin size for the density calculations (provided the bins were sufficiently small, e.g., $<0.25 \sigma)$.

\section{MECHANICAL PROPERTIES OF NANOSCOPIC STRUCTURES}

The nanoscopic structures investigated in this work were rectangular cantilever plates supported on a substrate. The 


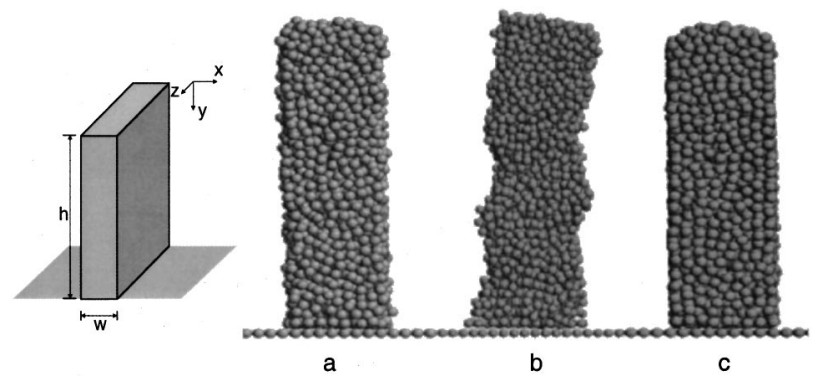

FIG. 3. Schematic representation and typical configurations of nanoscopic structures. Cross sections are shown for structures prepared using three methods: (a) etched with chain scission, (b) etched without chain scission, and (c) nano-imprinted.

setup closely resembles the lines of polymeric material that are involved in the manufacture of microcircuits. A schematic representation of these lines is shown in Fig. 3. These lines are reminiscent of free-standing films in that they have three free interfaces; only one facet of the lines is in direct contact with the substrate. Periodic boundary conditions were applied along the $z$ direction, resulting in infinitely long cantilever plates of height $h$ and width $w$.

Most of the structures considered here had aspect ratios

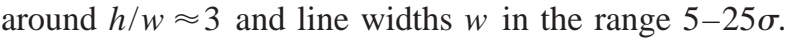

These structures are so small that, in actual practice, there is little flexibility in the methods employed to create them. Two fabrication techniques that are currently being considered are radiation lithography and flash-imprint lithography. In radiation lithography, a polymeric film is exposed to incident photons through a mask. Exposed regions of the material undergo a chemical reaction, which creates a physical or chemical differential throughout the film. In the particular case of poly(methylmethacrylate) (PMMA), a polymer that is used to fabricate prototype or model structures, the incident radiation has the effect of severing the backbone of the molecules, thereby reducing considerably the molecular weight of exposed regions. Low molecular weight material is more readily soluble in solvents, thereby offering a mechanism to dissolve selectively parts of the film. In the case of a chemically-amplified material, the radiation catalyzes a reaction which alters the chemical nature of side groups along the polymer chain, thereby rendering it more or less soluble in a specific solvent; the degree of polymerization of the molecules is not altered to a significant extent. In nano-imprint lithography, a mold is simply pressed onto a molten polymer film and the system is annealed. The mold is removed after cooling, leaving behind the desired pattern.

Given the simplicity of the model employed in this work, it is not possible to reproduce faithfully the fabrication processes employed in practice. The structure preparation methods considered below are only meant to provide a firstorder account of the main molecular features that arise as a result of specific fabrication techniques.

\section{A. Structure preparation}

\section{Preparation by chain scission}

The starting systems for the preparation of nanoscopic structures were supported films of different thicknesses, gen-
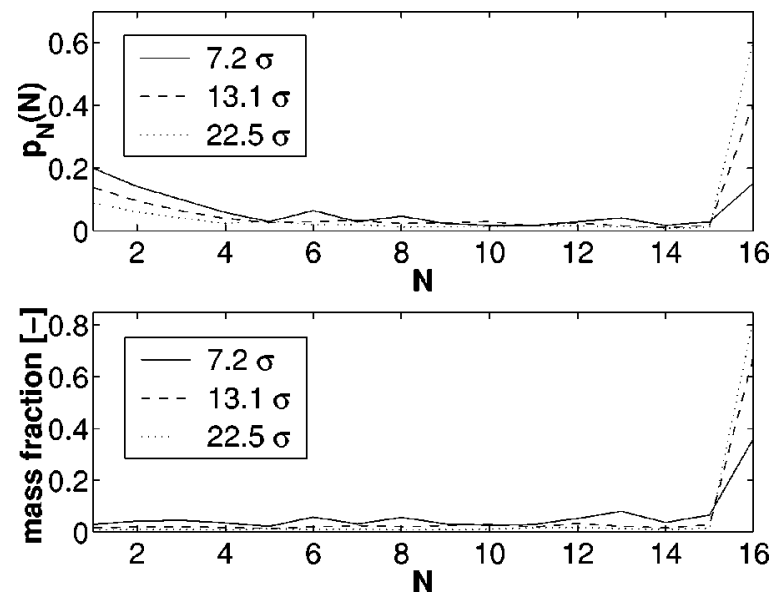

FIG. 4. Probability distribution (top) and mass-fraction distribution (bottom) as a function of chain length for three polymer nanostructures prepared by chain-scission. The width $w$ of the structures is provided in the inset.

erated according to procedures outlined above (in the context of the glass transition temperature). The films were gradually cooled down to a temperature of $T^{*}=0.20$, which is well below $T_{g, \text { bulk }}$.

In the next step, all monomers outside a threshold area were removed from the film, resulting in the desired platelike structures. Some of the bonds of the polymer chains were necessarily severed. This process resembles the fabrication of PMMA lines by exposure to x-rays.

After removing the particles, the remaining structures were placed on a substrate consisting of particles arranged on a quadratic (crystalline) lattice with grid size $\sigma$ [see Fig. 3(a)]. The particles used for the substrate were identical to the monomer units but were held fixed in space. (This step is necessary because smooth walls are not able to support forces parallel to the wall. This would result in the structures sliding across the surface when subjected to a bending force.) As the final preparation step, the structures were equilibrated for $t^{*}=5000$ at a temperature $T^{*}=0.20$. Note that equilibration at temperatures above $T_{g}^{*}$ led to flow and a "melting" of the structures.

Figure 3(a) shows a representative cross section of the structures obtained by chain-scission. This process leads to relatively sharp, smooth surfaces and a fairly regular geometry (this is also consistent with experiment; the surface roughness of PMMA lines is small).

Clearly, the resulting polymeric material is polydisperse. This is also true of experimental PMMA nanostructures, where the molecular weight of the material near the air interface is lower than in the bulk. Figure 4 shows the number and mass distribution functions for three different structures of widths $7.2,13.1$, and $22.5 \sigma$, respectively. For intermediate or large structures (e.g., 13.1 or $22.5 \sigma$ ), only a small fraction of the chains is influenced by the scission process.

\section{Preparation by chain dissolution}

In this preparation method the integrity of the chains is preserved; entire chains having a center-of-mass outside the threshold area are removed, thereby resulting in structures that are rougher and more irregular than those created by 
chain scission. This preparation technique resembles the exposure and dissolution process of chemically amplified materials.

After placing the resulting structures on the same substrate as that employed in the previous section [see Fig. $3(\mathrm{~b})]$, the systems were allowed to equilibrate for $t^{*}$ $=5000$. During the equilibration period, molecules near the surface of the structures rearrange to fill some of the small voids left behind by "dissolved" molecules. A typical configuration of a structure created in this manner is shown in Fig. 3(b).

\section{Nano-imprinting}

The third preparation method resembles nano-imprint lithography. To simulate this process, equilibrium configurations were generated in different sized boxes (representative of a mold) using the Expanded Grand Canonical Ensemble (EGCE) technique. ${ }^{20}$ These configurations were generated using a cubic lattice model; they were subsequently turned into square-well chains (in continuous space), and used as a starting point for extensive equilibration runs using DMD. To represent the mold, the side walls of the simulation box were modeled as smooth surfaces with a purely repulsive potential. The lower wall, which represents a substrate, was modeled as a smooth square-well wall. The upper side of the mold was left open to allow the structures to expand and contract freely. The configurations were then cooled starting from a temperature of $T^{*}=1.0$ and using a stepwise temperature ramp with decrements of $\Delta T^{*}=-0.04$. At each temperature, the systems were run for $t^{*}=100$. Note that our starting temperature of $T^{*}=1.0$ is well in the liquid regime, and the configurations lose all memory of the lattice in the initial stages of our calculations. After cooling the systems to the target temperature of $T^{*}=0.20$, the mold was removed and the structures were placed onto the same substrate used for the other types of structures (see sections above). The structures were further equilibrated for $t^{*}=5000$ before they were used for production simulations.

As can be seen in Fig. 3(c), this method leads to highly regular structures that exhibit a pronounced layering arrangement near the surfaces. The layering effect is unrelated to any remnant of the lattice starting configuration. In fact it grows during annealing, and it persists even after the mold is removed. To illustrate the magnitude of the layering, Fig. 5 shows the density profile of the structure along the $x$-axis after the equilibration. The strong layering near the free surface slowly disappears towards the inner region of the structures.

\section{B. Bending simulations}

To determine the apparent Young's modulus of nanoscopic structures, a constant line force was applied in the direction normal to the plates, along the top of a structure. Figure 6 illustrates schematically how the force is applied. The deflection of the structures in response to the applied force was determined as a function of time.

More specifically, a force was applied to a rod placed near the top of the structure via an acceleration term. The rod

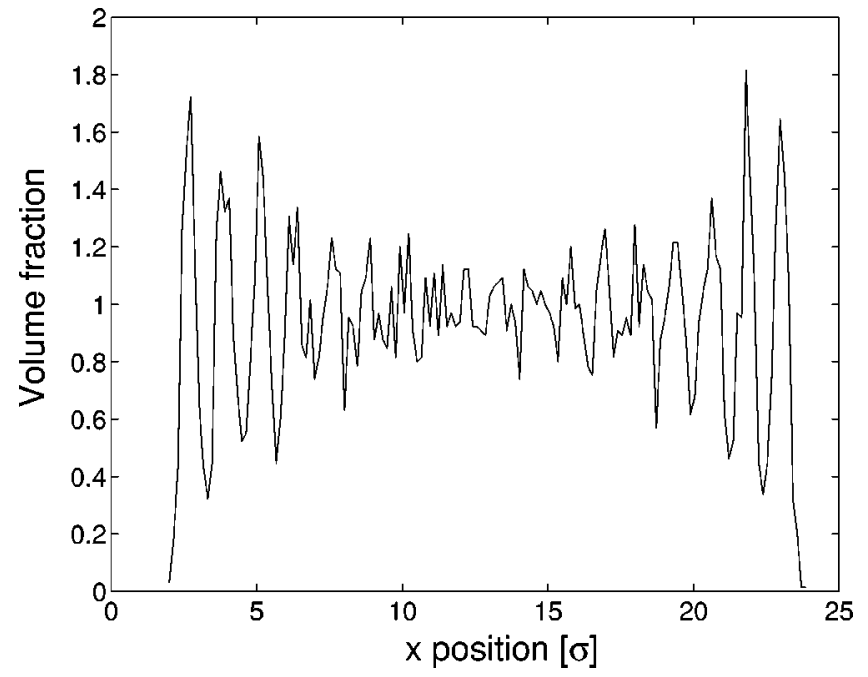

FIG. 5. Density profile of nano-imprinted structure along the $x$-axis.

consists of a linear arrangement of tangent hard spheres. The spheres are fixed relative to each other, and the rod acts as a single, rigid object. Each of the spheres has a diameter $\sigma$, and a total mass of 1000 monomer units. The ensuing deflection of a structure was measured by calculating the centerof-mass in a slab of thickness $2 \sigma$ parallel to the substrate and centered at the point of application of the force (see Fig. 6).

Figure 7 shows a plot of the deflection at the top of the structure as a function of time after application of the force. Initially, the deflection increases rapidly; it then reaches a steady-state value asymptotically. The figure also shows fast oscillations (corresponding to the natural frequencies of the structure) superimposed on the deflection curve. Note, however, that only the steady-state position is of interest to our analysis, and this was determined by averaging data over a time period ranging from $t^{*}=3000$ to $t^{*}=5000$. The time average was determined in ten blocks of length $t^{*}=200$, and the standard deviation of those blocks was used as an error estimate.

The sway beam model has been used in previous work to analyze the mechanical behavior of polymer lines. ${ }^{3}$ This model neglects the effects of lateral contraction due to a
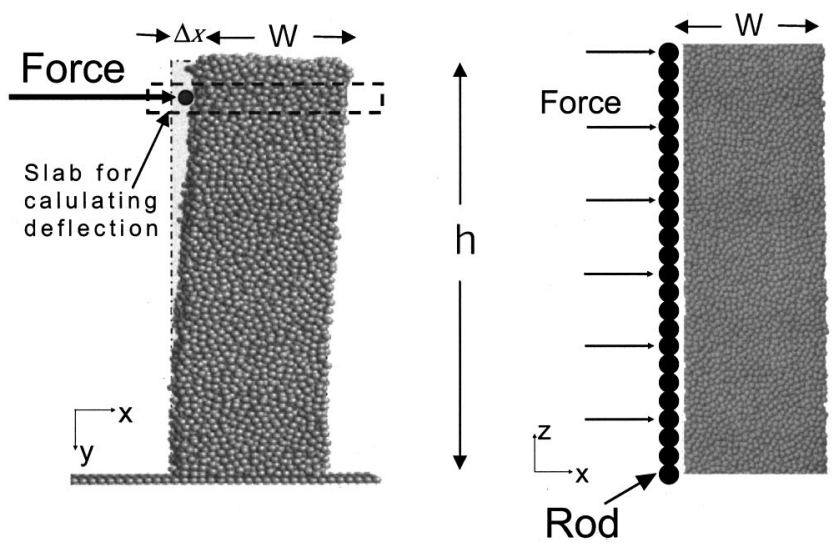

FIG. 6. Cross sectional view (left) and top view (right) of a bending simulation. 


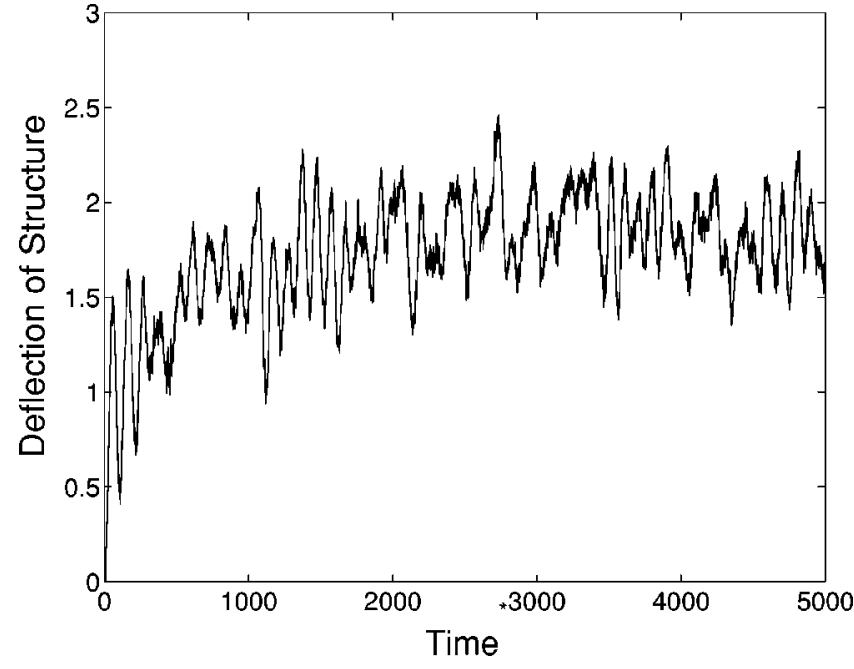

FIG. 7. Deflection at the top of a structure as a function of time, following the application of a lateral force.

nonzero Poisson's ratio. As the systems considered here are plates, rather than beams, a bending plate theory is more appropriate. According to component theory, the general solution for the elastic bending of long rectangular plates expressed in terms of the initial parameters is given by ${ }^{21}$

$$
\begin{aligned}
\delta x(y)= & \overline{\delta x}_{o}-\bar{\omega}_{y 0} y-\bar{m}_{y 0} \frac{y^{2}}{2 K} \\
& -\bar{q}_{y 0} \frac{y}{6 K}\left(y^{2}-\frac{6 w^{2}}{5(1-\nu)}\right),
\end{aligned}
$$

where $\delta x(y)$ is the deflection of the plate at point $y, \omega_{y 0}$ is the $y$ component of the rotation of the middle plane at $y$ $=0, m_{y 0}$ is the bending moment at $y=0, q_{y 0}$ is the shear force per unit length at $y=0$ normal to the plate, $\nu$ is Poisson's ratio, $w$ is the thickness of the plate, and $K$ is the flexural rigidity, given by

$$
K=\frac{E h^{3}}{12\left(1-\nu^{2}\right)} .
$$

Here, $E$ is the Young's modulus of the material.

The simulated structures can be viewed as cantilever plates clamped at the base and subject to a shear force at the top of the structure. Applying these boundary conditions yields the following expression for the deflection at the top of the plate,

$$
d_{0}=\frac{4 f\left(1-\nu^{2}\right)}{E}\left(\frac{h}{w}\right)^{3}\left[1+\frac{3}{5(1-\nu)}\left(\frac{w}{h}\right)^{2}\right] .
$$

Equation (5) is only valid in the linear or elastic regime, i.e., for small deformations. It is therefore important to ensure that the simulations are conducted in that regime. For a typical polymer at room temperature, plastic deformations start to arise at deflections larger than roughly $4 \%$ of the structure height. In order to avoid the plastic regime, the maximum forces for simulations were estimated in such a way as to cause at most a $\sim 3 \%$ deflection. Since the actual mechanical properties of a nanoscopic structure are not known a priori, the forces required to induce a $3 \%$ deflection

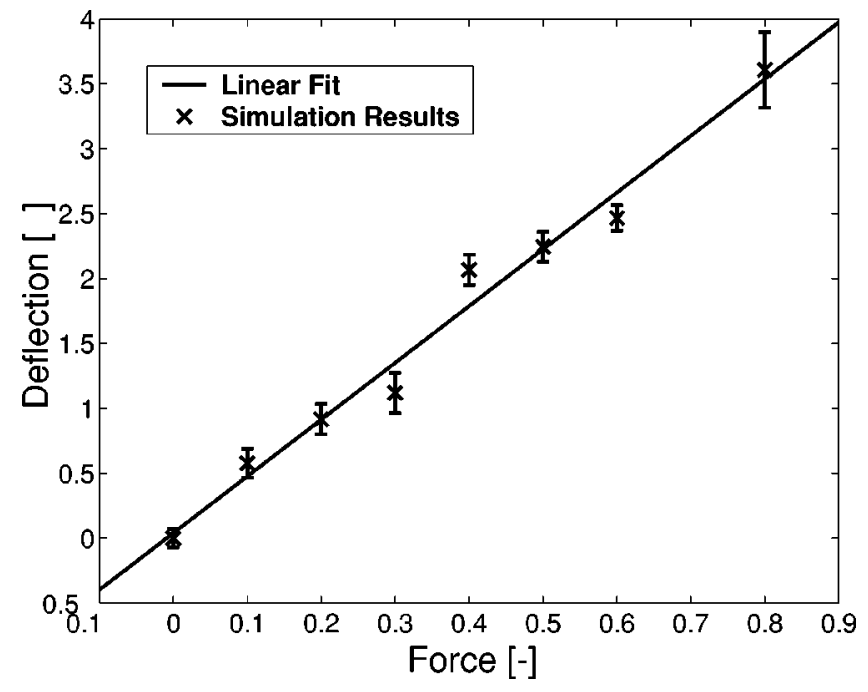

FIG. 8. Measured deflection as a function of applied force for a polymer structure of width $9 \sigma$, prepared by chain scission.

were estimated from the bulk value of the Young's modulus for the material at the same density. Multiple forces ranging from 0 to the maximum force were applied, and the corresponding deflections were measured. By performing multiple deflection simulations, it is possible to confirm that bending experiments are conducted in the linear regime, and the statistical quality of the deflection data is improved considerably.

Typical simulated deflection values are shown in Fig. 8 as a function of force. The data in that figure correspond to a structure having dimensions $9.0 \sigma \cdot 29.0 \sigma$, prepared via chain scission. The response is clearly linear, even for relatively large relative deflections. For the actual calculation of the Young's modulus, only forces up to $f^{*}=0.3$ were considered.

The slope of the stress-strain curve (Fig. 8 in this case) was determined from a linear least-squares fit. From the slope, the Young's modulus can be calculated according to Eq. (5). Use of that equation requires that the dimensions of the structure be determined in a systematic manner. This is complicated by the highly irregular shape of some of the structures, particularly those etched without chain scission. A consistent measure of the linewidth was achieved by calculating density profiles along the $x$-axis. In those density profiles, the boundaries of the structures were defined to be the points where the density of the material dropped below $50 \%$ of the average density of the structures. As only the centerof-mass of each spherical particle (or monomer) was used to calculate the density profiles, an additional $0.5 \sigma$ was added to the results on both sides (the particle diameter is $1 \sigma$ ). Determining the height of the structure is not necessary, as the quantity of interest is the point of application of the force, which is directly known from the simulations.

In addition to the dimensions, the Poisson's ratio of the material must be known in order to extract the Young's modulus from Eq. (5). Unfortunately, the Poisson's ratio of a nanoscopic structure is not known a priori, and we currently do not have a method at our disposal to calculate it from the simulations. However, physical bounds of this ratio are 
known. The maximum value that it can have is $0.5 .^{22}$ Furthermore, while negative values of Poisson's ratio have been reported, ${ }^{23,24}$ it can be safely assumed that in simple materials it is positive. (We note that one of the critical features for exhibiting negative Poisson's ratios is an asymmetry induced by lateral groups incorporated in the molecular backbone. This feature is absent in our materials, which consists of short and smooth linear polymer chains.) We find that Young's moduli estimated from Eq. (5) do not vary by more than $15 \%$ when using $\nu=0$ or $\nu=0.5$; for simplicity, in all of our calculations we use the bulk value, determined as described below.

\section{Alternative approaches}

As pointed out throughout this manuscript, the bending simulations are prone to errors and large statistical uncertainties. While special care has been exercised to conduct the modulus analysis in as systematic a manner as possible, the estimated errors associated with the calculation of the Young's modulus remain large.

Unfortunately, experimental data on the mechanical properties of nanoscopic structures are not available. The measurement of these properties is challenging, and while we are aware of several researcher's efforts in that area, no results have been produced to date. In the absence of data, we are left to verify that our predictions are at least internally consistent by using alternative numerical techniques. The following two alternative simulation methods were also implemented in this work.

\section{Frequency analysis}

As shown in Fig. 7, the deflection of a nanoscopic structure is accompanied by pronounced oscillations corresponding to the natural eigenfrequencies of the system. Those frequencies can be measured and used to determine the apparent Young's modulus of the structures. In order to do so, the structures were excited by an impulsive force applied to the top of the structure. The force was applied until a deflection of $0.5 \sigma$ was achieved.

Figure 9 shows the power spectral density of an impulse response for a structure having $w=9.0$ prepared by chain scission (the temperature was $T^{*}=0.20$ ). The spectral density was calculated using Welch's averaged periodogram method. ${ }^{25}$ One characteristic frequency dominates the response; apart from small high-frequency oscillations and noise, it is almost sinusoidal. It can be shown that this frequency is indeed the first eigenfrequency of the systems, as the oscillations of the deflection on different heights are all in phase. For higher eigenfrequencies, one would expect to see a phase shift at different positions along the structure. None of the higher eigenfrequencies are sufficiently clear, as the application of an impulsive force at the top of the structure mainly excites the first eigenmode. Furthermore, higher eigenfrequencies experience a higher dampening, and are difficult to analyze.

The eigenfrequencies of a structure depend on its geometry and the mass and mechanical properties of the structure. For a given geometry and a given mass, a vibration analysis can be used to determine the Young's modulus of the mate-

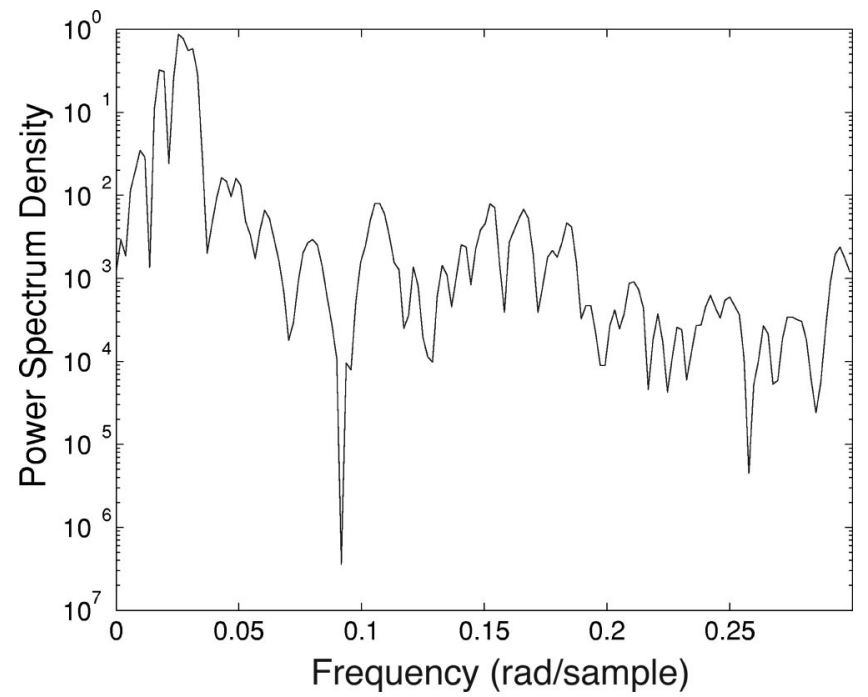

FIG. 9. Power spectral density of impulse response for a structure of width $9 \sigma$ prepared by chain scission.

rial. For a simple bending vibration of an infinitely long cantilever plate, the eigenfrequencies are given $b y^{26}$

$$
\omega_{i}(\mathrm{rad} / \mathrm{s})=\frac{\lambda_{i}^{2}}{h^{2}}\left(\frac{E w^{3}}{12\left(1-\nu^{2}\right) \rho^{*}}\right)^{1 / 2},
$$

where $\rho^{*}$ is the mass per unit area and $\lambda_{i}$ are the eigenvalues. For the first two modes, the eigenvalues are $\lambda_{1}$ $=1.87510407$ and $\lambda_{2}=4.69409113$. As before, the Poisson's ratio $\nu$ was set at the bulk value, namely 0.4 . Here, the error made by this assumption could at most be about $20 \%$.

\section{Constant-deflection method}

As a third means for determining the apparent Young's moduli, a different deflection technique (referred to as the "constant-deflection" technique) was used for the structures prepared without chain scission. In this method, a rod was used to impose a permanent deflection on the structure and was held at a fixed position. The forces exerted by the structure on the rod were then determined to construct force vs deflection curves. The apparent Young's modulus was extracted from such curves using the same model as for the bending simulations.

\section{Determination of bulk properties}

In order to establish whether the mechanical properties of a nanoscopic structure are different from those of the bulk, it is essential to determine the moduli of the bulk material for the model considered in this work. These constants were calculated using the elastic-bath method of Van Workum et al. ${ }^{27}$ which has been shown to provide efficient and accurate calculations of the elastic constants of amorphous glasses and crystalline materials. ${ }^{28}$ In this method, the size and shape of the simulation cell containing the bulk sample are allowed to fluctuate. ${ }^{29}$ The simulation cell is immersed in an elastic bath to improve the statistical accuracy of the calculation. The isothermal elastic-constant tensor is determined from the strain fluctuations according to 
TABLE I. Bulk values determined by the fluctuation method.

\begin{tabular}{ccccccc}
\hline \hline$T^{*}$ & $\begin{array}{c}\text { No. of } \\
\text { particles }\end{array}$ & $\begin{array}{c}\text { Volume } \\
\left(\sigma^{3}\right)\end{array}$ & $\begin{array}{c}\text { Pressure } \\
\left(\sigma^{-3} \epsilon\right)\end{array}$ & $\begin{array}{c}\text { Density } \\
\left(\sigma^{-3}\right)\end{array}$ & $\begin{array}{c}\text { Young's } \\
\text { modulus } \\
\left(\sigma^{-3} \epsilon\right)\end{array}$ & $\begin{array}{c}\text { Poisson's } \\
\text { ratio } \\
(-)\end{array}$ \\
\hline 0.20 & 926 & 910.8 & -0.483 & 1.017 & 39.0 & 0.40 \\
0.25 & 926 & 920.4 & -0.475 & 1.006 & 37.6 & 0.40 \\
0.30 & 926 & 928.9 & -0.425 & 0.997 & 31.3 & 0.37 \\
\hline \hline
\end{tabular}

$$
C_{i j k l}=\frac{k_{B} T}{V_{0}}\left[\left\langle\epsilon_{i j} \epsilon_{k l}\right\rangle-\left\langle\epsilon_{i j}\right\rangle\left\langle\epsilon_{k l}\right\rangle\right]^{-1}-\frac{V_{b}}{V_{0}} C_{i j k l}^{b},
$$

where $\epsilon_{i j}$ is the strain tensor, $k_{B}$ is the Boltzmann constant, $T$ is the temperature, and $V_{0}$ is the average volume; $C_{i j k l}^{b}$ and $V_{b}$ are the elastic constants and the volume of an elastic bath, respectively, which is used to improve the efficiency of the simulations. The indices represent the Cartesian coordinates in three dimensions. The strain tensor is given by

$$
\boldsymbol{\epsilon}_{i j}=\frac{1}{2}\left[\langle\widetilde{h}\rangle_{i k}^{-1} G_{k l}\langle h\rangle_{l j}^{-1}-\delta_{i j}\right] .
$$

If $\mathbf{a}, \mathbf{b}$, and $\mathbf{c}$ are the principal axes of the simulation box, then $h_{i j}=\{\mathbf{a}, \mathbf{b}, \mathbf{c}\}_{i j}$ describes its size and shape. The matrix $\langle h\rangle_{i j}$ describes the reference box and $\langle\widetilde{h}\rangle_{i j}^{-1}$ is the inverse of the transpose of $\langle h\rangle_{i j}$. The metric tensor $G_{i j}$ is defined as $G_{i j}=\widetilde{h}_{i k} h_{k j}$. The Young's modulus of the bulk sample is related to the coefficients of $C_{i j k l}$. In this work, $V_{b}=V_{0}$, while $C_{i j k l}^{b}$ was chosen to optimize the convergence of Eq. (7).

Initial bulk configurations were generated using DMD. The pressure was determined in such a way as to produce an average bulk density comparable to that observed in the inner region of the structures investigated in this work. The pressures determined were negative, as the structures show residual tensile stresses. These residual stresses originate in the periodic boundary conditions used during the cooling process of the films; films cannot contract freely in the direction of periodic boundary conditions, resulting in the observed residual tension. However, we note that real systems exhibit similar residual stresses as a free contraction of the surface layers is inhibited by the interaction with the substrate.

The bulk values obtained by this method are shown in Table I. The listed values for the Poisson ratio were used to determine the Young's modulus from Eq. (5).

\section{E. Results and discussion}

\section{Glass transition temperature}

The results for $T_{g}$ corresponding to the four types of films investigated in this work are shown in Fig. 10. A significant reduction of $T_{g}$ is observed for free-standing films at thicknesses below approximately $12 \sigma$. This decrease can be attributed to an enhanced segmental mobility near the free surface. For thin films, the interfacial region represents a large fraction of the overall system, thereby resulting in an overall increase in mobility.

In contrast, for all the supported and grafted films considered in this work, the $T_{g}$ of thin films increased above the bulk value. Interestingly, the critical size for the departure from bulk behavior appears to be the same for all four film types. The increase in $T_{g}$ is attributed to a reduction of segmental mobility in the vicinity of the substrate. As the reduction of mobility is stronger in the two surface grafted films, the increase in $T_{g}$ is even more pronounced.

The simulation results are in qualitative agreement with experiments. For free-standing films, the observed depression of $T_{g}$ is consistent with scattering data for polymer films. ${ }^{11}$ For supported films, the predicted increase in $T_{g}$ is consistent with ellipsometry data ${ }^{7}$ and local thermal probe data. ${ }^{10}$ For grafted films, the simulated increases in $T_{g}$ are consistent with the thermal probe data of Fryer et al., ${ }^{30}$ although the magnitude of the increases is not nearly as pronounced. These results suggest that even the material properties of the highly simplified polymer model employed in this work exhibit an intrinsic size dependence, which parallels some of the trends observed in real polymeric materials. It is therefore of interest to go beyond the study of the glass transition, and address the issue of whether the mechanical properties of this polymer model also depend on size. Mechanical-property data for ultrathin films or nanoscopic structures are not available; predictions using this simple model could provide useful insights.

\section{Mechanical properties}

Bending simulations were used to determine the apparent Young's moduli of nanoscopic structures in the range of line thicknesses from $\sim 5 \sigma$ to $\sim 25 \sigma$. Table II lists the dimensions and aspect ratios for all the structures considered in this work. Note that the aspect ratios are not uniform; this is

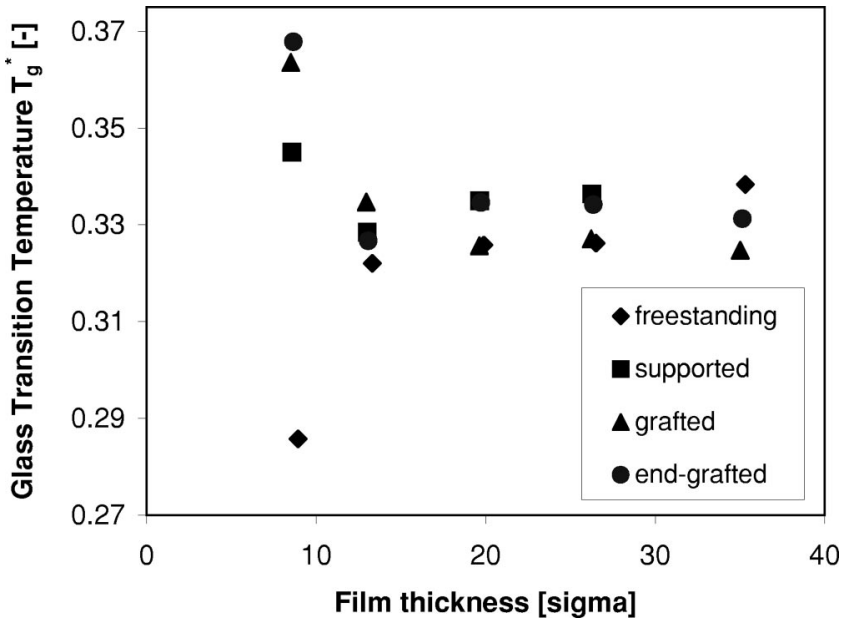

FIG. 10. Glass transition temperature as a function of film thickness for four different types of films. 
TABLE II. Dimensions and aspect ratios for structures used in this work.

\begin{tabular}{lccc}
\hline \hline \multicolumn{1}{c}{ Type } & $\begin{array}{c}\text { Linewidth } w \\
w\end{array}$ & $\begin{array}{c}\text { Height } h \\
\text { of application of force }\end{array}$ & $\begin{array}{c}\text { Aspect } \\
\text { ratio }\end{array}$ \\
\hline Chain scission & 4.6 & 11.0 & 2.39 \\
Chain scission & 7.2 & 19.0 & 2.64 \\
Chain scission & 9.0 & 29.0 & 3.22 \\
Chain scission & 13.1 & 39.0 & 2.98 \\
Chain scission & 17.2 & 59.0 & 3.43 \\
Chain scission & 22.5 & 79.0 & 3.51 \\
Chain dissolution & 5.0 & & 2.20 \\
Chain dissolution & 7.3 & 11.0 & 2.60 \\
Chain dissolution & 9.6 & 19.0 & 3.02 \\
Chain dissolution & 12.9 & 29.0 & 3.02 \\
Chain dissolution & 16.2 & 39.0 & 3.64 \\
Chain dissolution & 23.5 & 59.0 & 3.36 \\
Nano-imprinting & 7.5 & 79.0 & 2.07 \\
Nano-imprinting & 9.2 & 20.0 & 2.93 \\
Nano-imprinting & 13.2 & 27.0 & 3.11 \\
Nano-imprinting & 17.1 & 41.0 & 3.45 \\
Nano-imprinting & 19.5 & 59.0 & 3.23 \\
\hline \hline
\end{tabular}

due to the fact that the dimensions of a structure change during the initial equilibration period, and it is therefore difficult to anticipate with precision what the final aspect ratio will be.

In Fig. 11 results are shown for the apparent Young's moduli of structures prepared by chain scission. All results are for a temperature of $T^{*}=0.20$, and they are normalized with respect to the bulk value of the Young's modulus.

For the largest structure ( $w=22.5 \sigma)$, the Young's modulus extracted from bending simulations is in good agreement with that obtained from bulk strain fluctuations. The results for smaller structures exhibit significant departures from the bulk. For the smallest structures considered here, the apparent Young's modulus is five times smaller than that of the bulk material. Our simulations reveal a pronounced dependence of Young's moduli on size, with deviations from bulk behavior starting to arise at line widths of around $w \approx 20 \sigma$.

In Fig. 11 results are shown for the apparent Young's moduli of structures prepared by chain dissolution (without chain scission). While the actual values of the moduli differ

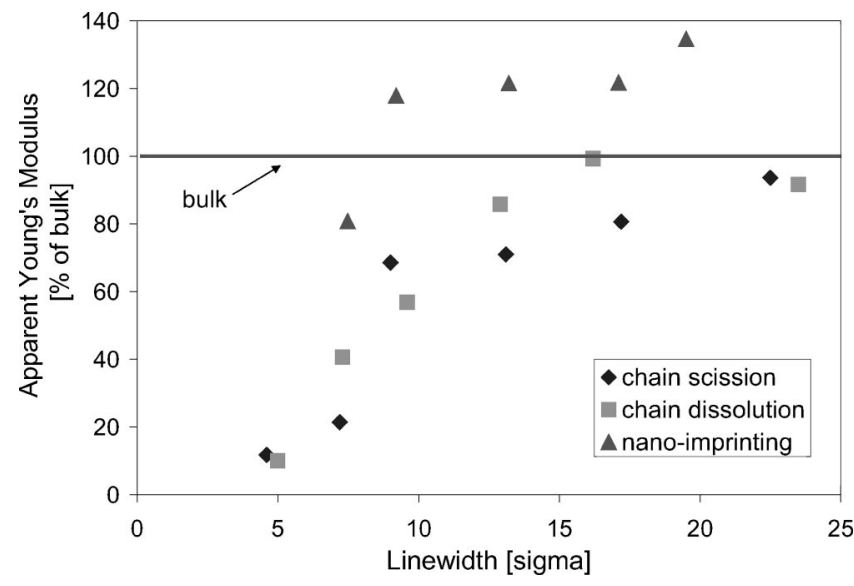

FIG. 11. Apparent Young's modulus for the three structure types (see Fig. 3) at $T^{*}=0.20$. slightly from those obtained for chain-scission structures, the overall behavior is comparable. For the largest structures, the apparent Young's modulus matches the bulk value, while small structures are significantly weaker.

In contrast, nano-imprinted structures exhibit a qualitatively different behavior. As can be seen in Fig. 11, the apparent moduli of small structures indicate a higher mechanical strength than in the bulk. Only the smallest structure shows a slight decay in the Young's modulus below the bulk value.

In order to examine the temperature dependence of the observed, size-induced changes of the Young's modulus, bending simulations for structures etched with chain scission were repeated at temperatures of $T^{*}=0.25$ and $T^{*}=0.30$, i.e., closer (but still below) the bulk glass transition temperature $\left(T_{g \text {, bulk }} \approx 0.33\right)$. Note that the bulk Young's modulus at $T^{*}=0.30$ is already $20 \%$ smaller than that at $T^{*}=0.20$ (see Table I). The bending simulations were conducted on the same structures as those employed earlier at $T^{*}=0.2$ (Fig. 11). The resulting Young's moduli are shown in Fig. 12. As before, they are normalized with respect to the bulk values at the corresponding temperatures. In both cases the departures from the bulk behavior set in at larger line widths and are generally more pronounced than at $T^{*}=0.20$. For $T^{*}$ $=0.30$, the apparent Young's modulus of the largest structure $(w \approx 22.5 \sigma)$ is a factor of 2 smaller than that of the bulk.

Figure 13 shows the apparent Young's moduli for a temperature of $T^{*}=0.2$ as determined by the frequency analysis. The results are normalized with respect to the result obtained for the largest structure etched with chain scission (note that we do not have a method for determining the bulk moduli from a frequency analysis). The results from the frequency analysis are consistent with those obtained from bending experiments; the structures prepared by chain scission exhibit a decay of the apparent Young's moduli with decreasing size, while nano-imprinted structures show an increased mechanical strength. 


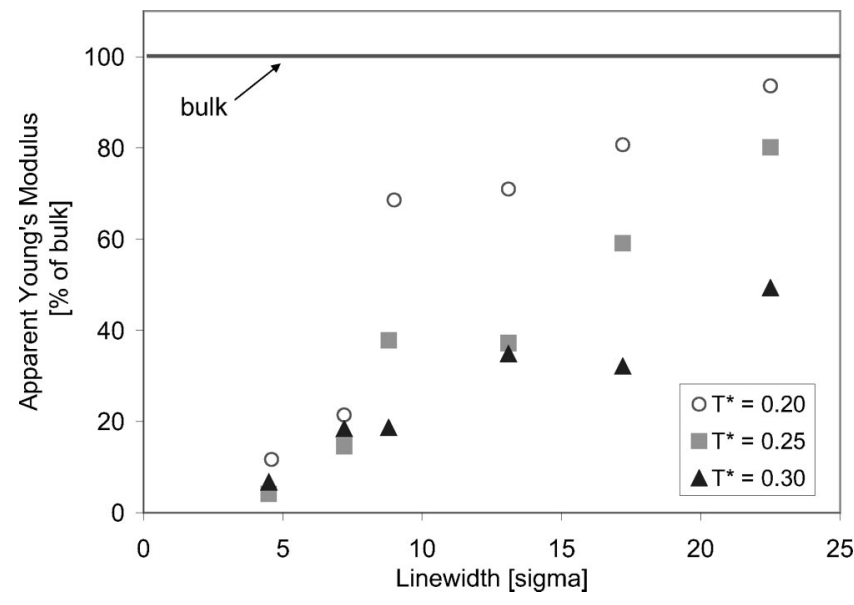

FIG. 12. Apparent Young's modulus for structures etched with chain scission at higher temperatures.

Consistent with the bending calculations and the frequency analysis, the results obtained by the "constantdeflection" technique show a strong decrease in the apparent Young's modulus with decreasing line width of the structure. The results obtained from the alternative methods lend credence to the validity of our predictions, and suggest that the results reported here are not an artifact of a particular simulation technique, but a direct consequence of the small dimensions of our nanoscopic structures.

Our simulations for both excised or chemicallyamplified polymeric structures exhibit a size-induced decrease in the mechanical strength. Part of the reduction in the Young's modulus for the excised structures could be attributed to the fact that the polymer near the air interface has a lower molecular weight than the bulk (see Fig. 4). While the fraction of low molecular-weight material is relatively small $(\approx 8 \%$ for the structures having line width of $22.5 \sigma)$ simulations (or experiments) cannot separate molecular-weight effects from small-length-scale effects. Note that real PMMA polymeric structures are actually prepared in a manner that alters the molecular weight and resembles our simulations. Furthermore, the loss of mechanical strength is still signifi-

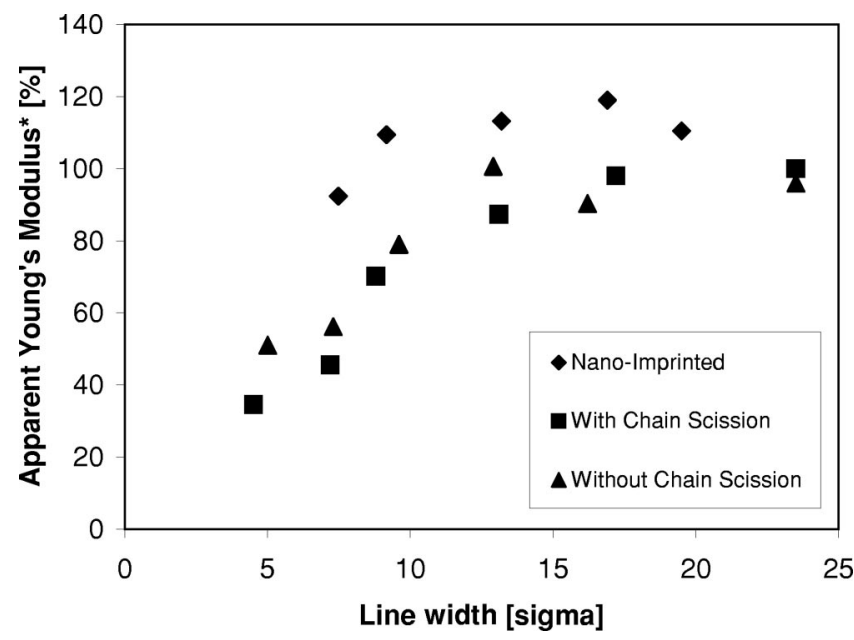

FIG. 13. Apparent Young's modulus determined by frequency analysis. cant for the structures prepared without chain scission, where the molecular weight is not altered.

Many of the simulations were conducted at a temperature of $T^{*}=0.2$, which is well below $T_{g \text {, bulk }} \approx 0.33$. The decay of the Young's modulus, however, is comparable in magnitude to that observed when a bulk polymeric material is heated above $T_{g}$. As shown above, the $T_{g}$ of ultrathin freestanding polymer films decreases with decreasing thicknesses. As discussed earlier, this depression of $T_{g}$ can be traced back to a greater mobility of the molecules near the air interface. The nanoscopic structures considered here resemble free-standing films. The molecular mobility near the air interface is such that significant stress relaxation can occur in the interfacial region. This is clearly revealed by the molecular strain analysis discussed below. Of course, as the dimensions of a structure decrease, the fraction of material in the interfacial region increases significantly, thereby changing the overall mechanical behavior of the system.

These effects become increasingly pronounced for temperatures closer to $T_{g}$, as can be seen from the results at $T^{*}=0.25$ and $T^{*}=0.30$. For both temperatures, even the largest structures considered here are significantly weaker than the bulk. Unfortunately, at near- $T_{g}$ temperatures we are unable to simulate structures large enough to recover bulk behavior (the computational demands of larger structures are beyond our current resources). Assuming a simple linear decay of the apparent Young's modulus, the critical size limit for the departure from the bulk can be estimated to be $\sim 27 \sigma$ for $T^{*}=0.25$ and $\sim 46 \sigma$ for $T^{*}=0.30$.

In contrast to etched structures, nano-imprinted structures exhibit an enhancement of mechanical stability compared to the bulk. This increased mechanical strength is due to a reorganization of the molecules upon annealing in a mold, which gives rise to layered, quasicrystalline arrangements (see Fig. 5).

\section{Molecular strain analysis}

The coupling between structure bending and local, molecular events, can be investigated in more detail by calculating the molecular strain distribution of nanoscopic structures. For small, elastic deformations, the molecular strains are small except near the air interface, where mobility is enhanced. More interesting observations can be made during plastic deformations, where a few, isolated molecular events dictate the final state of a nanoscopic structure.

The local, molecular strain tensor was calculating according to a procedure described in the literature. ${ }^{31}$ This method employs Delaunay triangulations to divide the system into tetrahedra, each spanning four neighboring particles. The strain tensors for each tetrahedron can then be calculated ${ }^{32}$ they are associated with the interstices between the particles, rather than the particles themselves. In Ref. 31, the strains were therefore reallocated proportionally to the particles using Voronoi polyhedra. This step was omitted in this work, as the systems considered are dense and the strain tensors associated with the interstices are expected to bear the same information as the reallocated, "atomic" strains. In our analysis, the strain tensors, averaged over the $z$-coordinate, were associated with the closest grid point in a 

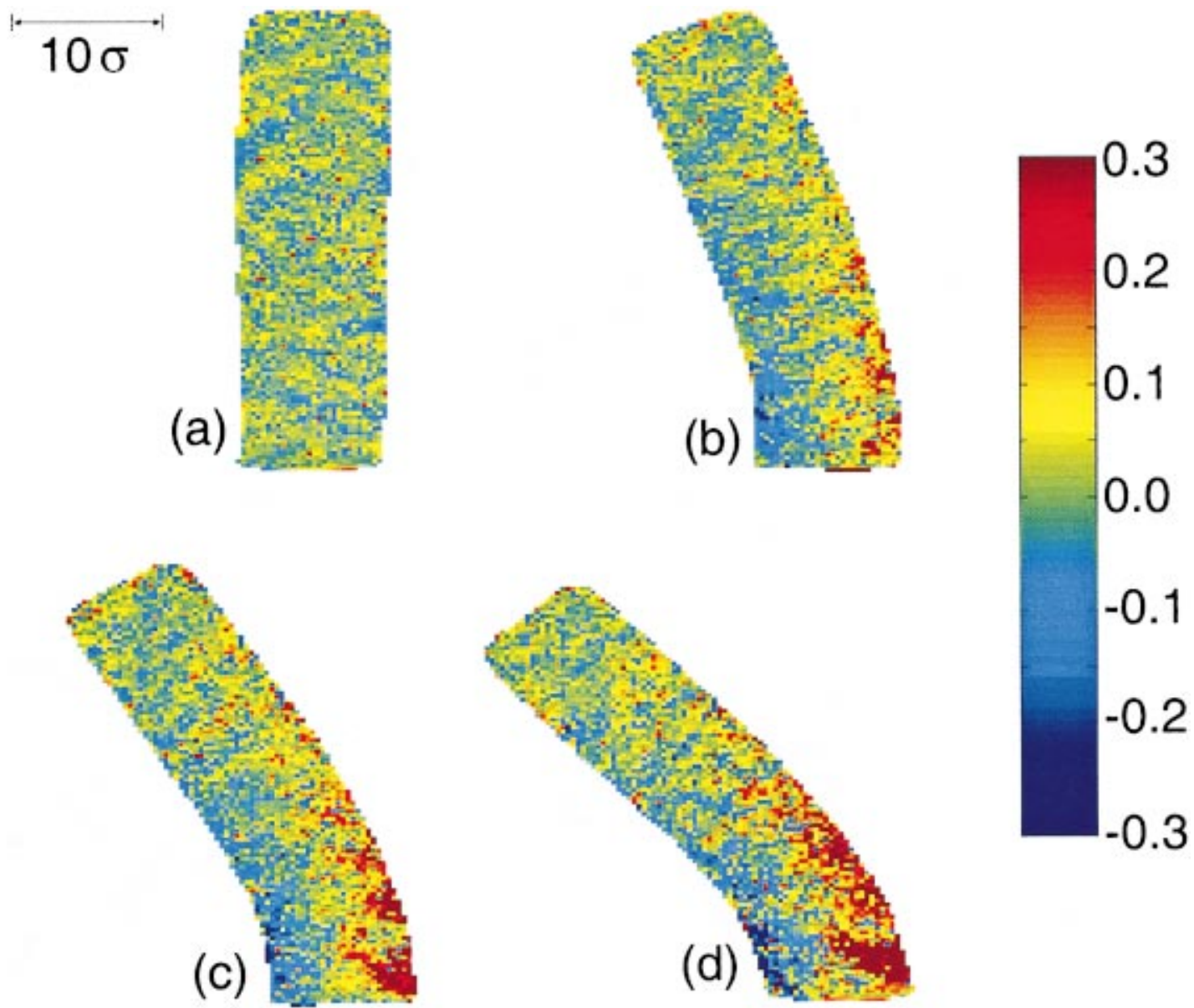

FIG. 14. (Color) Cross-sectional view of the molecular strains ( $y y$-component) that arise during plastic deformation of a structure of width $9 \sigma$. The four snapshots correspond to four stages along the deformation process.

two-dimensional grid with a spacing of $0.25 \sigma$. In the case of multiple associations to the same grid point, average values were employed.

To study the plastic deformation of a nanoscopic structure, the analysis was conducted using a structure prepared by chain scission and a linewidth of $9 \sigma$, at a temperature of $T^{*}=0.20$. At time $t^{*}=0$, a force was applied to the top of

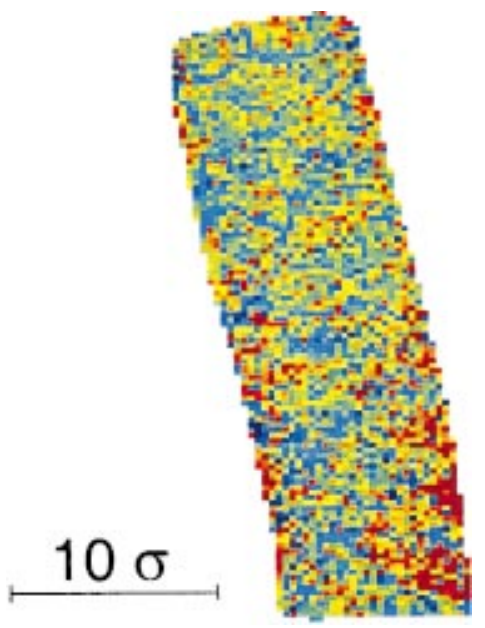

FIG. 15. (Color) Cross section of a structure after plastic deformation showing the $y y$-component of the residual molecular strain. the structure, thereby causing a large, plastic deformation. The instantaneous strain distributions inside the structure were calculated throughout the deformation process.

The $y y$-component of the molecular strain tensor is shown in Fig. 14 for four snapshots of the system, each corresponding to different deflection stages. The first snapshot shows the strain distribution in the undeformed state. The overall strain is neutral (mostly green), with short-lived local strain fluctuations. While these fluctuations can sometimes

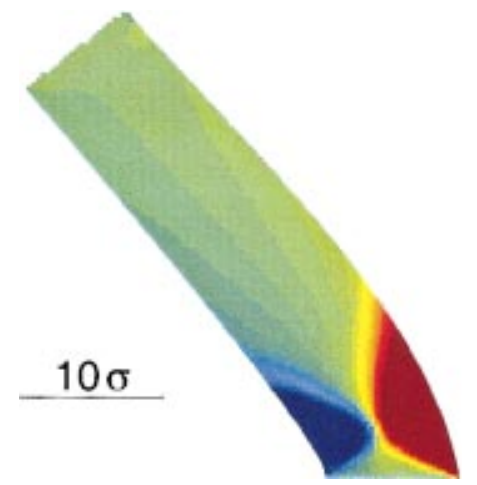

FIG. 16. (Color) Cross section of a structure after plastic deformation showing the $y y$-component of the steady-state strain distribution, as determined by continuum mechanics using the elasto-plastic stress-strain relation with parameters corresponding to PMMA. 
be considerable, particularly near the free interface, they are limited to small regions and do not persist over long periods of time.

In the second snapshot [Fig. 14(b)], the same structure is shown as the bending progresses. On the right side, tensile regions begin to form along the free surface of the structure, while compression is observed on the opposite side. The tensile regions appear to be highly localized, and concentrated to several small kernels, rather than being distributed contiguously along the edge of the structure. The regions of highest strain are located at the base of the structure, where the stresses are maximal.

As time progresses [Fig. 14(c)], the tensile regions propagate deeper into the structure in a nearly triangular shape. The propagation of these high-strain regions proceeds in a "fingering" manner, with intercalated regions of zero strain. This effect is particularly evident near the base of the structure. As one moves up along the edge of the structure, the regions of high strain slowly disappear; the top half of the structure is unstrained, aside for fast molecular fluctuations.

The state of maximum deflection is shown in Fig. 14(d). The highly localized, high-strain region near the base of the structure propagates even further into the system. This region of the structure is likely to yield and provide the onset of a crack. Note, however, that in our simulations the actual formation of a crack was never observed. This could be due to several reasons. First, the structures considered here are relatively small $(w=9 \sigma)$. Second, our polymer model does not contemplate the possibility of bond breaking. In real polymers, bond breakage can be an important mechanism in the formation of cracks. ${ }^{33}$ Furthermore, as the mobility in the surface regions is enhanced, the material might plastically deform and relieve stresses before they exceed the critical value for fracture.

Plastic deformation occurs in the regions of high molecular strain. This is further emphasized by the fact that, after removing the bending force and allowing the structure to relax, the resulting configuration exhibits significant residual strains, Fig. 15, localized around the base of the system. Even in the case of residual strains, the deformations are confined to a few small areas of the structure; regions of tensile strain clearly exhibit most of the residual deformations.

It is of interest to compare the results of our molecular strain analysis to the strain distribution arising from a continuum description of the system. To that end, finite element calculations of structure bending were performed on a structure having similar characteristics. Note, however, that the material was assumed to be homogeneous. A simple twodimensional plane strain model was employed for these calculations, which were conducted using ANSYS. ${ }^{34}$ In direct analogy to our molecular simulations, a force was applied at the top of a rectangular structure. A simplified elasto-plastic stress-strain relation was adopted, reminiscent of that observed experimentally in PMMA. ${ }^{35}$ This relation comprises an elastic regime and a yield regime. In the elastic region, the relation between stress and strain is linear, with a slope correponding to the Young's modulus ( $E=3 \mathrm{GPa}$ for PMMA at $30^{\circ} \mathrm{C}$ ). For stresses above a yield stress of $\sigma_{\text {yield }}=30 \mathrm{MPa}$, plastic deformation was modeled by reducing the slope of the stress-strain curve to $1 / 1000$ of the Young's modulus. The aspect ratio of the structure and the ultimate deflection were chosen to be comparable to those obtained in molecular simulations.

Figure 16 shows the steady-state solution for the $y y$-strain distribution of a deflected structure. The results reveal several qualitative differences with molecular simulations. In contrast to the highly localized regions of high strain observed in simulations, continuum calculations predict a single, large region of high strain. Furthermore, the continuum-mechanics region of high compression penetrates halfway into the structure, while our molecular analysis indicates that compressed regions are limited to a small region near the free interface.

\section{CONCLUSIONS}

Previous experimental ${ }^{11}$ and modeling ${ }^{12,14}$ work has shown that the glass transition temperature of thin polymer films depends on their thickness. The results presented here go beyond the glass transition, and provide numerical evidence in support of a departure of the mechanical properties of polymer nanoscopic structures from bulk behavior. Nanoscopic structures excised from amorphous, glassy polymer films exhibit a loss of mechanical strength. The critical dimension below which these effects become apparent is temperature dependent. For the lowest temperature considered in this work $\left(T^{*} / T_{g}=0.6\right)$, the departures from bulk behavior set in below line widths of approximately $\sim 20 \sigma$ (the aspect ratio is approximately 3 ); at temperatures in the vicinity of the glass transition, the critical size for departures from the bulk is estimated to be in the range of up to $\sim 46 \sigma$. In contrast, nanoscopic structures prepared by annealing in a mold (nano-imprinting) exhibit a slight increase of mechanical strength. This increase is attributed to a molecular layering effect that arises during annealing, giving rise to highly ordered, quasicrystalline structures.

A molecular strain analysis revealed pronounced qualitative differences between molecular bending simulations and continuum finite-element calculations. At the small length scales considered in this work, inhomogeneities in the material play an important role in determining the mechanical behavior of the overall structures; our results suggest that several assumptions of continuum mechanics are no longer justified at these length scales. More specifically, our continuum calculations were performed under the assumption that nanoscopic structures are homogeneous; in light of the results presented here, a logical next step would be to use simulated, local values for the stress and density fluctuations as an input for continuum models. We are pursuing such calculations using more refined molecular models, and the results of that work will be presented in the future.

It is of interest to relate the quantities obtained by our simulations to actual physical length and energy scales. If we view the interaction sites of our model as representative of a polymer Kuhn length, then $\sigma \approx 2 \mathrm{~nm}$. This results in critical dimensions for departures from bulk behavior of approxi- 
mately $40 \mathrm{~nm}$ at temperatures well below $T_{g}$. Near $T_{g}$, departures are likely to occur at longer length scales, in the neighborhood of $80 \mathrm{~nm}$. We emphasize again that, in view of the simplicity of the model considered in this work, these estimates should be considered with caution. They do provide, however, well-founded evidence in support of a loss of mechanical strength at dimensions of relevance to the semiconductor industry.

More generally, the results of this work serve to underline the fact that structure-property relations at nanoscopic dimensions can be complex and are poorly understood. Molecular simulations can help us elucidate some of these complex relations, but it is essential that experimental methods be devised in order to determine if and to what extent our predictions are valid; we hope that the work presented here will motivate such experiments.

\section{ACKNOWLEDGMENTS}

This work was supported by the Division of Chemical Sciences, Office of Sciences, U.S. Department of Energy, and by a grant from the Semiconductor Research Corporation (No. 2002-MJ-985).

${ }^{1}$ The International Technology Roadmap for Semiconductors, http:// public.itrs.net.

${ }^{2}$ H. B. Cao, P. F. Nealy, and W.-D. Domke, J. Vac. Sci. Technol. B 18, 3303 (2000).

${ }^{3}$ T. Tanaka, M. Morigami, and N. Atoda, Jpn. J. Appl. Phys., Part 1 32, 6059 (1993).

${ }^{4}$ A. Kaul, A. Gangwal, and S. S. Wadhwa, Curr. Sci. 76, 1561 (1999).

${ }^{5}$ A. Kawai, J. Vac. Sci. Technol. B 17, 1090 (1999).

${ }^{6}$ U. Laudahn, S. Fähler, H. Krebs, A. Pundt, U. v. H. M. Bicker, and U. Geyer, Appl. Phys. Lett. 74, 647 (1999).

${ }^{7}$ J. L. Keddie, R. A. Jones, and R. A. Cory, Europhys. Lett. 27, 59 (1994).

${ }^{8}$ J. L. Keddie, R. A. Jones, and R. A. Cory, Faraday Discuss. 98, 219 (1994).
${ }^{9}$ W. E. Wallace, J. H. Van Zanten, and W. L. Wu, Phys. Rev. E 52, R3329 (1995).

${ }^{10}$ D. Fryer, P. Nealey, and J. J. de Pablo, Macromolecules 33, 6439 (2000).

${ }^{11}$ J. A. Forrest, K. Dalnoki-Veress, and J. R. Dutcher, Phys. Rev. E 56, 5705 (1997).

${ }^{12}$ J. A. Torres, P. F. Nealy, and J. J. de Pablo, Phys. Rev. Lett. 85, 3221 (2000).

${ }^{13}$ P. Doruker and W. L. Mattice, Macromolecules 40, 4685 (1999).

${ }^{14}$ T. S. Jain and J. J. de Pablo, Macromolecules 35, 2167 (2002).

${ }^{15}$ N. Kenkare, S. W. Smith, C. K. Hall, and S. A. Khan, Macromolecules 31, 5861 (1998).

${ }^{16}$ A. V. Smith and C. Hall, J. Chem. Phys. 113, 9331 (2000).

${ }^{17}$ B. J. Alder and T. E. Wainwright, J. Chem. Phys. 31, 459 (1959).

${ }^{18}$ D. C. Rapaport, J. Phys. A 11, L213 (1978).

${ }^{19}$ S. W. Smith, C. K. Hall, and B. D. Freeman, J. Comput. Phys. 134, 16 (1997).

${ }^{20}$ J. J. de Pablo and F. Escobedo, J. Chem. Phys. 105, 4391 (1996).

${ }^{21}$ V. Panc, Theories of Elastic Plates (Noordhoff International Publishing, Leyden, 1975).

${ }^{22}$ R. Hibbeler, Mechanics of Materials (MacMillan, New York, 1991).

${ }^{23}$ L. Gibson, M. Ashby, G. Schajer, and C. Robertson, Proc. R. Soc. London, Ser. A A382, 25 (1982).

${ }^{24}$ C. He, P. Lui, and A. Griffin, Macromolecules 31, 3145 (1998).

${ }^{25}$ P. Welch, IEEE Trans. Audio Electroacoust. AU-15, 70 (1967).

${ }^{26}$ A. W. Leissa, Vibration of Plates, NASA Technical Report, Vol. SP-160, 1969.

${ }^{27} \mathrm{~K}$. Van Workum and J. J. de Pablo (unpublished).

${ }^{28}$ K. Van Workum, R. Faller, and J. J. de Pablo (unpublished).

${ }^{29}$ M. Allen and D. Tildesley, Computer Simulation of Liquids (Clarendon, Oxford, 1987).

${ }^{30}$ D. Fryer, R. Peters, E. Kim, J. Tomaszewski, J. J. de Pablo, P. Nealey, C. White, and W. Wu, Macromolecules 34, 5627 (2001).

${ }^{31}$ P. H. Mott, A. S. Argon, and U. W. Suter, J. Comput. Phys. 101, 140 (1992).

${ }^{32}$ M. Parinello and A. Rahman, J. Chem. Phys. 76, 2662 (1982).

${ }^{33}$ H. H. Kausch, Polymer Fracture, 2nd ed. (Springer-Verlag, New York, 1987).

${ }^{34}$ Ansys, Inc., http://www.ansys.com

${ }^{35}$ E. M. Arruda, M. Boyce, and R. Jayachandran, Mech. Mater. 19, 193 (1995). 